INVESTIGACIONES

\title{
Aprendizaje cooperativo y tutoría entre iguales en entornos virtuales universitarios*
}

\author{
Cooperative learning and peer tutoring in virtual university environments \\ Aprendizagem cooperativa e tutoria entre pares em ambientes virtuais universitários
}

\author{
Natalia González Fernández, ${ }^{a}$ Rosa García Ruiz, ${ }^{b}$ Antonia Ramírez García. ${ }^{c}$ \\ a Departamento de Educación, Área MIDE, Facultad de Educación, Universidad de Cantabria, España. \\ Fono: 0034-942201270. Correo electrónico: gonzalen@unican.es \\ b Departamento de Educación, Área DOE, Facultad de Educación, Universidad de Cantabria, España. \\ Fono: 0034-942201174. Correo electrónico: garciarm@unican.es \\ c Departamento de Educación, Área MIDE, Facultad de Educación, Universidad de Córdoba, España. \\ Fono: 0034-957212600. Correo electrónico: ed1ragaa@uco.es
}

\begin{abstract}
RESUMEN
La enseñanza universitaria tiende a crear más espacios virtuales de aprendizaje y a impulsar metodologías para mejorar el proceso de enseñanza-aprendizaje. Este trabajo pretende demostrar la potencialidad de la tutoría entre iguales, las herramientas 2.0 y el aprendizaje cooperativo para desarrollar conocimiento y competencias socioprofesionales en estudiantes universitarios en entornos virtuales, en diferentes titulaciones de la Universidad de Cantabria. Los objetivos son conocer en profundidad el alcance del proceso de innovación educativa propuesto, y saber cómo valoran los estudiantes la utilidad de las herramientas 2.0, el aprendizaje colaborativo y la tutoría entre iguales. La metodología cualitativa aplicada consiste en implementar un trabajo cooperativo virtual y evaluar resultados con un Focus Group. Los resultados revelan que el alumnado comprende los conceptos metodológicos y teóricos propuestos, y desarrolla competencias socioprofesionales relacionadas con la autorregulación, el pensamiento reflexivo y crítico, el saber, saber hacer y saber estar. Se identifican categorías clave tras la experimentación.
\end{abstract}

Palabras clave: aprendizaje cooperativo, tutoría entre iguales, educación superior, herramientas 2.0.

\begin{abstract}
University education tends to create more virtual learning spaces and promotes methodologies to enhance the teaching-learning process. This work aims to demonstrate the potentials of peer tutoring, web 2.0 tools, and cooperative learning to develop knowledge and skills in college students of socio virtual environments, in different degrees of the University of Cantabria. The objectives are to learn in depth the scope of the educational innovation process proposed and to know what is the value given by students to 2.0 tools, collaborative learning, and peer tutoring. A qualitative methodology is applied to implement a virtual collaborative work and evaluate results in a Focus Group. The results show that students understand the methodological and theoretical concepts proposed and developed skills related to socio-professional self-regulation, reflective and critical thinking, knowledge, expertise and know how to be. Key categories are identified after the experiment.
\end{abstract}

Key words: cooperative learning, peer tutoring, higher education, 2.0 tools.

El presente trabajo forma parte de un Proyecto de Innovación Docente (PID) titulado: "La tutoría entre iguales a través de las TIC y las metodologías participativas para la mejora del aprendizaje en educación superior", dirigido por la Dra. Natalia González Fernández. Ha sido financiado por el Vicerrectorado de Calidad e Innovación de la Universidad de Cantabria, España (1 $1^{\mathrm{a}}$ convocatoria, 2011-2012). 


\section{RESUMO}

O ensino universitário tende a criar mais espaços virtuais de aprendizagem e a impulsionar metodologias para melhorar o processo de ensino-aprendizagem. Este trabalho objetiva demonstrar a potencialidade da tutoria entre pares, as ferramentas 2.0 e a aprendizagem cooperativa para desenvolver conhecimentos e competências socioprofissionais em estudantes da Universidade de Cantabria, de diferentes titulações. Os objetivos são conhecer em profundidade o alcance do processo de inovação educativa proposto e conhecer como os estudantes valorizam a utilidade das ferramentas 2.0, a aprendizagem colaborativa e a tutoria entre pares. A metodologia qualitativa aplicada consiste na implementação de um trabalho cooperativo virtual e avaliação dos resultados com um Grupo Focal. Resultados mostram que os estudantes compreendem os conceitos teóricos e metodológicos propostos e desenvolvem competências socioprofissionais relacionadas com autorregulação, pensamento reflexivo e crítico, saber, saber fazer e saber estar. Depois da experimentação, são identificadas categorias chave.

Palavras chave: aprendizagem cooperativa, tutoria entre pares, ensino superior, ferramentas 2.0.

\section{INTRODUCCIÓN}

El nuevo sistema universitario demanda tanto del profesorado como del alumnado la implementación de nuevas metodologías de enseñanza-aprendizaje. Por un lado, se requiere del docente un rol de guía, supervisor y dinamizador del proceso de aprendizaje de los estudiantes, y por otro lado, a éstos se les exige un rol más activo y participativo dentro y fuera del aula. Todo ello conlleva el desarrollo de las denominadas metodologías activas.

Nuestra propuesta innovadora trata de acercarse a la tutoría entre iguales, las herramientas $2.0 \mathrm{y}$ el aprendizaje cooperativo como estrategias para transmitir saberes y competencias socioprofesionales en educación superior, proponiendo dos ejes fundamentales para lograr la mejora del aprendizaje de los estudiantes: utilizar las herramientas 2.0 para procesar, compartir, generar y gestionar la información y el conocimiento entre profesorado y estudiantes, e implementar la Tutoría entre Iguales como metodología que favorezca la adquisición de competencias socioprofesionales como la motivación intrínseca, el pensamiento reflexivo y crítico, las habilidades comunicativas y sociales, la autorregulación y la autoplanificación hacia su propio aprendizaje. Estas competencias, según la Comisión para la Educación del Siglo XXI (UNESCO, 1996) y $\operatorname{DeSeCo}^{1}$ (2002), son defendidas como sustanciales y ya son contempladas por diferentes titulaciones universitarias en nuestro país.

A lo largo del curso 2011-2012, los tres profesores que han diseñado este proyecto se han encargado de implementar, realizar el seguimiento y evaluar la tutorización entre iguales, implicando alumnado de diferentes asignaturas y titulaciones de la Facultad de Educación de la Universidad de Cantabria. Tomando como referente los beneficios derivados del trabajo cooperativo en entornos virtuales, que lo convierten en un indicador plausible y predictor de los buenos resultados exigidos en las sociedades plurales y participativas (Camilli, López Gómez y Barceló, 2012; Yuste, Alonso y Blázquez, 2012; Gozálvez Pérez, Traver Martí y García López, 2011; Hernando, Aguaded y Tirado, 2011) planteamos a nueve estudiantes, que a priori no se conocían, la oportunidad de trabajar distribuidos en tres equipos cooperativos heterogéneos para resolver un problema educativo real, utilizando como vehículo herramientas 2.0 (Blog, Google Docs/Drive, Google +, Twitter), pero más concretamente, creando su propia red personal de aprendizaje, la cual se erigió en instrumento clave de su aprendizaje y en la adquisición de las competencias socioprofesionales citadas.

Programa de la OCDE para la definición y la selección de competencias. 
El blog se articula como un espacio de referencia en el que los docentes publican la guía del trabajo a realizar, el calendario-agenda, la documentación y otros recursos online para orientar al alumnado en la propuesta. El alumnado, por su parte, lo utiliza para publicar sus opiniones, aportaciones y resolución final. De esta manera, quedan recogidas en Internet y su acceso es fácil y permanente, favorenciendo así el seguimiento por parte de todos los implicados en el proyecto. Twitter es una red de microbloging, fácilmente accesible por telefonía móvil, que potenció la comunicación e interacción rápida entre compañeros mediante mensajes cortos. Y Google Docs/Drive es una aplicación orientada al trabajo colaborativo en Internet que facilitó a los estudiantes trabajar tanto de forma síncrona como asíncrona, desde el aula o desde sus casas. En experiencias similares (Erstad, Gilje y Arnseth, 2013; Molina, Jimeno, Pérez-Samaniego, Devís-Devís y Villamón, 2013; Fernández Tilve y San Juan, 2012), se demuestra que las herramienatas 2.0 pueden ser un valioso instrumento para promover competencias como el trabajo autónomo de los estudiantes, y que interconectar las prácticas y el uso de estas herramientas en entornos académicos y extraacadémicos sigue siendo un reto.

Se trata pues de un proyecto desarrollado bajo un enfoque constructivista e interactivo de organización del trabajo fuera y dentro del aula, en el cual los estudiantes son responsables de su aprendizaje y del de sus compañeros, en una estrategia de corresponsabilidad para alcanzar metas e incentivos grupales. Nuestra pretensión es lograr la mejora del aprendizaje de los estudiantes, en cuanto a la adquisición de contenidos, el desarrollo de competencias en entornos virtuales y la potenciación de la metacognición, de modo que sean conscientes de las ventajas que la tutoría entre iguales les aporta a la hora de mejorar su aprendizaje. Se entiende así que la tutoría entre iguales es un método de aprendizaje cooperativo basado en la creación de parejas, con una relación asimétrica (derivada del rol de tutor o de tutorado a desempeñar, respectivamente), con un objetivo común, compartido y conocido (adquisión de una competencia), que se logra a través de una relación planificada por el docente (Duran y Vidal, 2004).

En el sistema universitario tradicionalmente se ha concedido más importancia a la enseñanza que al aprendizaje, de manera que el docente era el responsable de la asignatura y la transmisión de conocimiento, pero autores como Collis y Moonen (2011), Michavila (2009) y Gros y Romañá (2004) afirman que esta labor debe ser complementada, dado que los nuevos planteamientos del EEES exigen una mayor interactividad con y entre los estudiantes. La tutoría entre iguales cuenta con una dilatada aplicación en el ámbito anglosajón (Evans \& Moore, 2013; Topping, Dehkinet, Blanch, Corcelles \& Duran, 2013; Di Tommaso, 2012). Igualmente, experiencias como las de Arco y Fernández (2011), Fernández, Arco, Ortega y Heilborn (2011), Alzate-Medina y Peña-Borrero (2010), Álvarez y González (2005) y Lobato, Arbizu y del Castillo (2004) en España demuestran que con su aplicación se produce un incremento del rendimiento académico, una reducción de la tasa de fracaso, una mejora de habilidades sociales, de autorregulación del trabajo académico, de actitudes positivas hacia el estudio y un alto nivel de satisfacción hacia el aprendizaje.

En este artículo se exploran los procesos de producción iniciales y finales de una iniciativa llevada a cabo a lo largo de un curso escolar completo 2011-2012. A través del estudio de las dificultades y los progresos de este proyecto, hemos identificado dimensiones clave y códigos de conducta que los estudiantes universitarios ponen en juego para la resolución de los conflictos surgidos al desarrollar trabajo cooperativo y tutoría entre iguales a través de herramientas 2.0. Los objetivos de este trabajo se concretan en conocer 
en profundidad el alcance del proceso de innovación educativa propuesto, y en saber cómo valoran los estudiantes la utilidad de las herramientas 2.0, el aprendizaje colaborativo y la tutoría entre iguales en entornos virtuales.

\section{MATERIAL Y MÉTODOS}

La puesta en práctica de nuestro trabajo se centra básicamente en un proceso de investigación-acción, es decir, una reflexión constante entre la teoría y la práctica en la que se construye y comparte entre todas las personas participantes los descubrimientos obtenidos, conformando así una comunidad de aprendizaje "en red" y fomentando una cultura de trabajo cooperativo y participativo. Para ello, se hizo necesario organizar la planificación de una serie de fases en las que concretar las actuaciones a realizar, resumidas en la Tabla $\mathrm{N}^{\circ} 1$ :

Tabla $N^{\circ} 1$. Fases del proyecto de innovación

\begin{tabular}{|c|c|c|}
\hline $\begin{array}{c}1^{\text {a }} \text { FASE. } \\
\text { Aspectos estructurales }\end{array}$ & $\begin{array}{l}2^{\mathrm{a}} \text { FASE. } \\
\text { Desarrollo del proyecto }\end{array}$ & $\begin{array}{l}3^{\mathrm{a}} \text { FASE. } \\
\text { Valoración del proyecto }\end{array}$ \\
\hline $\begin{array}{l}\text { Actualización de conoci- } \\
\text { mientos y uso de herra- } \\
\text { mientas } 2.0 \text {. Talleres y } \\
\text { actividades de formación } \\
\text { del profesorado y del } \\
\text { alumnado participante. }\end{array}$ & $\begin{array}{l}\text { Seminario inicial: "Objetivos y finalidades } \\
\text { del trabajo cooperativo". Fomento inicial de } \\
\text { convivencia entre componentes del grupo. } \\
\text { Organización del proceso de coordinación y } \\
\text { tutorización entre asignaturas implicadas y } \\
\text { fomento de la participación de los grupos. } \\
\text { Organización del trabajo interactivo, donde } \\
\text { los alumnos son responsables de su } \\
\text { aprendizaje y el de sus compañeros. División } \\
\text { del grupo grande de } 9 \text { personas, en grupos } \\
\text { de } 3 \text { personas. } \\
\text { Consignas de actuación. Con este protocolo } \\
\text { debenplanificarlastareasconsensuadamente. } \\
\text { El trabajo de los pequeños grupos se } \\
\text { comparte y contrasta virtualmente con el } \\
\text { resto de los compañeros y los profesores } \\
\text { mediante el asesoramiento de todos. }\end{array}$ & $\begin{array}{l}\text { Evaluar el impacto del } \\
\text { proyecto en los estudian- } \\
\text { tes implicados mediante } \\
\text { un Focus Group. }\end{array}$ \\
\hline
\end{tabular}

Fuente: elaboración propia.

\subsection{DISEÑO, TÉCNICA E INSTRUMENTO}

En este trabajo nos posicionamos desde el paradigma cualitativo. Pretendemos aproximarnos al problema para comprenderlo a partir de datos proporcionados por los estudiantes universitarios participantes, considerando sus puntos de vista como relevantes y dignos de estudio. 
Nos servimos de un diseño metodológico en el que aplicamos como técnica cualitativa fundamental el Focus Group. Dicha técnica se caracteriza por proporcionar un método de exploración, que permite a los participantes expresar sus preocupaciones y/u opiniones dentro de un contexto que es útil para la comunidad científica (Zeller \& Carmines, 1980). La opción exploratoria fue la adoptada en este trabajo porque permite tanto identificar y estructurar nuevas preguntas de investigación surgidas dentro del grupo y valoradas como puntos importantes, como obtener directrices para investigaciones futuras (Angell \& Klassen, 1999).

Se seleccionó una muestra, mediante un muestreo de tipo intencional, de estudiantes de la Facultad de Educación de la Universidad de Cantabria. El criterio de selección fue que hubiesen participado previamente en el desarrollo del Proyecto de Innovación propuesto por los autores. El tamaño del grupo se decidió siguiendo las consideraciones de Merton, Fiske \& Kendall (1990) para garantizar la participación de todos los componentes y asegurar un abanico amplio de perspectivas. En total participaron 8 estudiantes más un moderador o coordinador, además de los otros dos profesores del proyecto, que registraron la información. En la Tabla $\mathrm{N}^{\circ} 2$ se describe la composición de los participantes del grupo:

Tabla $N^{\circ}$ 2. Perfil de los participantes del Focus Group

\begin{tabular}{|c|c|c|c|c|}
\hline & Sexo & Edad & Titulación & Código \\
\hline 1 & Masculino & 42 & Máster & C \\
\hline 2 & Femenino & 26 & Máster & E \\
\hline 3 & Masculino & 22 & Máster & N \\
\hline 4 & Femenino & 20 & $3^{\circ}$ Magisterio E.I. & M \\
\hline 5 & Femenino & 21 & $3^{\circ}$ Magisterio E.F. & CR \\
\hline 6 & Masculino & 38 & $3^{\circ}$ Magisterio E.F. & A \\
\hline 7 & Femenino & 21 & $3^{\circ}$ Magisterio E.I. & S \\
\hline 8 & Masculino & 21 & $3^{\circ}$ Magisterio E.F. & AM \\
\hline
\end{tabular}

Fuente: elaboración propia.

La duración no superó la hora y media. Se usó un guión temático para la moderación que fue poco directiva. Su contenido fue validado mediante la Técnica Delphi, por un grupo de 5 expertos de diferentes universidades españolas (Cantabria, Córdoba y Almería). El guión final se estructuró en 3 dimensiones, compuestas por 5 y 6 cuestiones, siguiendo las sugerencias de Krueger (2000), quien propone un número menor de diez (a menudo, alrededor de cinco o seis). Las preguntas fueron de naturaleza no estructurada de manera que los entrevistados pudieran contestarlas desde una amplia variedad de puntos de vista. En la siguiente Tabla $\mathrm{N}^{\circ} 3$ se presenta una síntesis del guión del Focus Group: 
Tabla $N^{\circ}$ 3. Guion Focus Group

\begin{tabular}{|c|c|}
\hline $\begin{array}{c}\text { HERRAMIENTAS } 2.0 \\
\text { EN EL } \\
\text { APRENDIZAJE } \\
\text { COOPERATIVO } \\
\text { VIRTUAL }\end{array}$ & $\begin{array}{l}\text { 1. ¿Qué rol cumplen las herramientas } 2.0 \text { en una enseñanza presencial? } \\
\text { ¿Y en una no presencial? } \\
\text { 2. ¿Estas herramientas han potenciado vuestra motivación intrínseca } \\
\text { hacia el aprendizaje en este proyecto? } \\
\text { 3. ¿Qué herramientas } 2.0 \text { han contribuido a desarrollar competencias de } \\
\text { pensamiento reflexivo y crítico, habilidades comunicativas, habilidades } \\
\text { sociales, autorregulación y autoplanificación? } \\
\text { 4. ¿Qué limitaciones os han condicionado para utilizar las herramientas } \\
\text { 2.0 en el proyecto? } \\
\text { 5. ¿Qué destacas positivamente del trabajo cooperativo virtual con las } \\
\text { citadas herramientas? } \\
\text { 6. ¿Qué propuestas de mejora propondrías para mejorar el trabajo on-line } \\
\text { y las reuniones virtuales? }\end{array}$ \\
\hline $\begin{array}{l}\text { TUTORÍA ENTRE } \\
\text { IGUALES DE } \\
\text { DIFERENTE } \\
\text { TITULACIÓN Y } \\
\text { EXPERIENCIA }\end{array}$ & $\begin{array}{l}\text { 1. ¿Cómo organizáis habitualmente el desarrollo de tareas en la } \\
\text { universidad: esperáis que os pauten los grupos, trabajáis con } \\
\text { compañeros/as conocidos/as, individualmente? } \\
\text { 2. ¿Cómo creéis que afecta a la calidad del trabajo ser tutorizado por un/a } \\
\text { compañero/a? } \\
\text { 3. ¿Qué consecuencias ha tenido en la elaboración del trabajo propuesto } \\
\text { en este proyecto tener que considerar las consignas de un/a } \\
\text { compañero/a? } \\
\text { 4. ¿Cómo contribuye a la elaboración del trabajo la libertad de actuación } \\
\text { respecto al tiempo, momentos y lugares de reunión? } \\
\text { 5. ¿Qué competencias socioprofesionales son necesarias para desarrollar } \\
\text { una tutoría entre iguales? }\end{array}$ \\
\hline $\begin{array}{l}\text { APRENDIZAJE } \\
\text { COOPERATIVO }\end{array}$ & $\begin{array}{l}\text { 1. ¿Qué significa aprender cooperativamente? } \\
\text { 2. ¿Creéis que los contenidos, actividades, consignas y recursos de un } \\
\text { proyecto han de estar completamente desarrollados por el docente? } \\
\text { 3. ¿Cómo valoráis el reto de trabajar desde la incertidumbre y de forma no } \\
\text { directiva? } \\
\text { 4. ¿Te has arriesgado proponiendo ideas o soluciones trabajando } \\
\text { cooperativamente? (¿habrías actuado igual si lo hubieras hecho solo/a? } \\
\text { ¿qué cambia?) } \\
\text { 5. ¿Se puede mejorar el aprendizaje trabajando cooperativamente? } \\
\text { 6. Inconvenientes y posibilidades de trabajar cooperativamente. }\end{array}$ \\
\hline
\end{tabular}

Fuente: elaboración propia.

El discurso producido en el grupo fue grabado en audio y video y luego transcrito textualmente, para ser analizado con la ayuda del software de procesamiento de datos cualitativos AtlasTi 7. En esta primera etapa de análisis cualitativo se siguieron las pautas propuestas por la teoría fundamentada, identificando las categorías iniciales a través de la codificación abierta (Strauss y Corbin, 2002).

El procedimiento analítico que plantea la Grounded Theory (Glaser \& Straus, 1999) se caracteriza por seguir una secuencia no lineal (Creswell, 1998), donde se parte desde los 
datos hacia un análisis que gira constantemente entre la organización, la interpretación y la teorización sobre éstos. El objetivo final es crear categorías teóricas a partir de los datos y analizar las relaciones relevantes entre ellas para construir una interpretación analítica del mundo de los participantes (Charmaz, 2005).

Posteriormente a las sesiones de audición y transcripción del Focus Group, se llevó a cabo un proceso de lectura analítica de los datos y una codificación primaria estableciendo comparaciones en búsqueda de similitudes y diferencias. La aparición reiterada de analogías y la generación de conceptos de mayor grado de abstracción, hizo posible la generación de una nueva codificación más explícita con la que se diseñó un sistema de categorías. La elaboración teórica inductiva se llevó a cabo con la ayuda del AtlasTi 7, desarrollando todo el trabajo posterior de recodificación, jerarquización y subsunción teórica de códigos en familias.

El proceso de análisis de contenido con AtlastTi 7 ha transcurrido en 4 etapas. Éstas son: comprensión de la transcripción y codificación, categorización, análisis y creación de redes entre categorías, e interpretación analítica.

\section{RESULTADOS Y ANÁLISIS}

Como resultado final de la codificación abierta se identificaron varias categorías iniciales y sus propiedades. Entre ellas, las más relevantes son las que se describen a continuación.

\subsection{CATEGORÍA PERCEPCIÓN DE UTILIDAD DE LAS HERRAMIENTAS 2.0 PARA DESARROLLAR UN TRABAJO COOPERATIVO A TRAVÉS DE LA TUTORÍA ENTRE IGUALES}

Se refiere al rol que los estudiantes creen que deben cumplir las herramientas 2.0, sobre todo en lo referente al desarrollo del aprendizaje cooperativo entre iguales. Esta categoría se generó en función de las posibilidades para reunirse todos los miembros de un grupo de forma presencial, bien para tutorizarse o para avanzar en el desarrollo de un trabajo, puesto que esa condición mediatizaba en gran medida qué, con quién, cuándo y cómo desarrollar una tutoría y un trabajo cooperativo. Las principales propiedades de la categoría son:
a) Percepción de funcionalidad.
b) Sensación de cercanía e inmediatez.
c) Impresión de ser un complemento.
d) Valoración de su eficacia.

Las herramientas 2.0 son concebidas por el alumnado no como una alternativa sino como un complemento multifuncional, que favorece una comunicación más cercana y plural. En este sentido, destacan el rol de apoyo educativo, de ser una fuente de contactos sin límites. Así lo expresa un estudiante entrevistado:

Es un grupo de herramientas que puedes utilizar para infinidad de cosas. Ahora cada vez casi para cualquier cosa. Y que te sirven para establecer una comunicación más cercana con los compañeros, para buscar datos y sitios de manera conjunta mucho más eficiente, ya no sólo 
centrándote en un aula o biblioteca de la universidad. Y también como sistemas de apoyo para tu aprendizaje, hay muchas herramientas que te ayudan a entender, si no es con textos es con videos $(\mathrm{N})$.

\subsection{CATEGORÍA APRECIACIÓN DE COMPETENCIAS DESARROLLADAS EN EL TRABAJO COOPERATIVO Y LA TUTORÍA ENTRE IGUALES A TRAVÉS DE HERRAMIENTAS 2.0}

Esta categoría representa las destrezas, capacidades y conocimientos que inciden de manera directa en el desarrollo óptimo de un trabajo cooperativo y tutorial entre iguales en entornos virtuales. Las principales propiedades de esta categoría son:
a) Motivación intrínseca.
b) Autoplanificación.
c) Autocontrol.
d) Habilidades sociales.
e) Espíritu de superación.
f) Saber hacer, saber estar, saber (dominio técnico).
g) Implicación.
h) Escucha activa.
i) Reflexión.
j) Pensamiento crítico.
k) Empatía.
1) Asertividad.

Los estudiantes inciden en que saber (dominar técnicamente) la aplicación o herramienta es fundamental para que sea un vehículo o canal eficaz de comunicación. Creen que no compromete la herramienta sino el saber hacer y saber estar de sus compañeros. Reconocen que las herramientas facilitan evidencias y contenidos, pero que el proceso y el producto final depende de la implicación y organización de todos los componentes de un grupo, y para ello es fundamental saber sintonizar diferentes ritmos, actitudes y comportamientos, es decir, dominar una serie de habilidades sociales y saber elegir el medio adecuado para hacer llegar la información al otro.

Si es a través de videoconferencias sí se facilita el uso de habilidades sociales. Si es a través de herramientas escritas, como Google Docs/Drive se pierde mucho, porque pierdes la riqueza del lenguaje no verbal, de la interacción, o de contenidos que si no los entiendes, no sabes por dónde está yendo el otro; puede estar haciendo una aportación constructiva y tú te lo estás tomando como una crítica negativa "me está diciendo que lo estoy haciendo todo mal", y la pretensión tan sólo era hacerme un comentario para aportar más a que el trabajo vaya por otro lado (A).

\subsection{CATEGORÍA LIMITACIONES DE TRABAJAR LA COOPERACIÓN Y LA TUTORÍA ENTRE IGUALES CON HERRAMIENTAS 2.0}

Describe los sucesos o situaciones generadas por el uso de herramientas 2.0 que han condicionado de forma insatisfactoria el desarrollo óptimo del trabajo cooperativo y la tutoría entre iguales. Las principales propiedades de la categoría son: 
a) Apreciación de dificultades técnicas.

b) Restricciones de expresión.

Los estudiantes entrevistados, en general, se sienten satisfechos con el uso académico que han podido hacer de las herramientas 2.0, a pesar de manifestar salvedades en relación a la poca capacidad de expresión que permiten herramientas como Twitter, lo que da lugar a malinterpretación o incomprensión del sentido del mensaje, y problemas con las conexiones al utilizar el Google + o el Google Docs/Drive. Uno de los entrevistados así lo manifiesta:

Con respecto a Google + o Goolge Docs/Drive, tecnológicamente el audio y el video daba errores, dificultades de conexión con el software, pero principalmente quizá lo chocante son las malas interpretaciones que se pueden dar por los pocos caracteres que se pueden escribir en Twitter (C).

\subsection{CATEGORÍA FACTORES QUE PUEDEN MEJORAR EL DESARROLLO DE UN TRABAJO COOPERATIVO Y LA TUTORÍA ENTRE IGUALES EN ENTORNOS VIRTUALES}

Se trata de plantear alternativas capaces de solventar las dificultades o imprevistos registrados en el uso académico de las herramientas 2.0 en concreto, con la finalidad de facilitar la cooperación y tutorización entre iguales. Las principales propiedades de la categoría son:

a) Necesidad de entrenamiento grupal.

b) Afrontamiento en solitario.

c) Gestión de incertidumbre, compromiso, autonomía y presión.

Se aprecia una discordancia entre la elevada valoración que dicen tener de las herramientas 2.0 y la escasa destreza que tienen a la hora de aplicarlas para redactar, compartir, debatir documentos y comunicarse con sus compañeros. En los inicios del trabajo cooperativo y tutorial se saturan, no están acostumbrados a ocuparse "del otro", a sintonizar distintos ritmos de aprendizaje y conocimientos. Este sentir se manifiesta en el Focus Group, donde exponen que:

Yo creo que, inicialmente, habría que hacer una fase de trabajar un poco más las herramientas conjuntamente entre los miembros del grupo, porque si no empiezas el trabajo, se te va echando el tiempo encima y tienes que estar explicando a un compañero una aplicación que no entiende, otro compañero no entra porque no sabe cómo solucionar un tema de audio (C).

Y que no es lo mismo que te lo expliquen en clase e inmediatamente utilizarlo, que estar en casa y decir "joder estoy solo y tengo que hacerlo". Por ejemplo te dices "esta pantallita que sale ahora ¿qué quiere decir? ¿Cuál es la solución? (CR).

Por ejemplo con el Google +, te das cuenta de que es muy "intuitivo", pero con la presión de que te espera un compañero para que le expliques, a las 21:00 hrs., sin poder conectar, llegando tarde, ¡la presión es esa! (E). 


\subsection{CATEGORÍA PREFERENCIAS PARA AGRUPARSE EN EL TRABAJO COOPERATIVO Y LA TUTORÍA ENTRE IGUALES EN ENTORNOS VIRTUALES}

Explica la forma y justificación en la que los estudiantes prefieren desarrollar un reto académico, bien individualmente, bien en un grupo con miembros conocidos y elegidos motu proprio, bien en agrupamientos pautados por los docentes. Las principales propiedades de la categoría son:
a) Aprendizaje previsible.
b) Aprendizajes múltiples.

Una constante en el Focus Group es la percepción disyuntiva entre lo positivo de elegir los componentes del grupo de trabajo, dado que se mantienen vínculos de confianza y seguridad, y lo positivo de trabajar en grupos pautados por los docentes, en los que se aprende a resolver conflictos, a tolerar, a compensar, a ser asertivo. Así es como lo pone de relieve uno de los estudiantes:

Trabajar en grupos pautados me gusta por la diversidad, el reto de poder conseguir atraer a alguien que en principio no quiere trabajar. Cuando puedo elegir prefiero hacer grupos mixtos, me gusta si el grupo es amplio, que tenga un determinado número de componentes conocidos, pero antepongo traer a alguien que sea diferente, porque sé que siempre aprendes algo. Como tengo mis limitaciones clarísimas, intento compensar el grupo, atraer a gente que seguramente tiene adquirida una competencia que a mí me falta, así, si puedo, la voy a intentar adquirir y nos vamos a intentar complementar, porque a todo no llegas. ¿Qué pasa cuando trabajas con grupos ya conocidos? Que sabemos sin necesidad de asignar roles concretos lo que cada uno asumirá (E).

\subsection{CATEGORÍA REPERCUSIÓN DE LA TUTORÍA ENTRE IGUALES EN EL APRENDIZAJE UNIVERSITARIO}

Refiere la trascendencia formativa de desarrollar feedback entre compañeros y las competencias que afectan a la conexión entre iguales hasta conseguir dar sentido a los aprendizajes. Las principales propiedades de la categoría son:
a) Debate fluido.
b) Asimilación inmediata.
c) Motivación.
d) Metodología.

Los estudiantes entrevistados se sienten escuchados y motivados por sus iguales, influye la cercanía y confianza a la hora de plantear dudas y "comprender antes", a pesar de manifestar cierta desconfianza respecto a la calidad y profundidad del aprendizaje y cierta decepción respecto al valor institucional que se le otorga a la tutoría entre iguales, entendiendo que se infravalora, malinterpreta y se apoya con una metodología docente magistral. Algunos de los estudiantes así lo declaran:

Al ser tutorizado por un compañero no es exactamente donde se aprende más, sino que estás más motivado trabajando con un compañero. Si estás al mismo nivel, por ejemplo en la misma clase, 
la motivación va a ser mayor porque nos enfrentamos al mismo reto y uno puede complementar al otro. Pero, también es cierto que si te está apoyando un profesor o un profesional, sabes que vas a poder avanzar más $(\mathrm{C})$.

La tutoría entre iguales no está valorada. Creo que se confunde mucho, no se ha dado el salto a rentabilizarla $(\mathrm{E})$.

He aprendido que todavía se puede esforzar uno más de ver a tus compañeros cómo se esfuerzan por enseñarte $(M)$.

\subsection{CATEGORÍA PARTICIPACIÓN COOPERATIVA DE ESTUDIANTES EN ENTORNOS VIRTUALES}

Recoge las sensaciones de los estudiantes cuando afrontan la resolución de un trabajo cooperativo basándose en sus investigaciones, cuando un trabajo constituye el "todo" de la asignatura, lo que finalmente se va a evaluar, teniendo en cuenta el proceso de aprendizaje y el producto. Implica el tránsito del rechazo a la incertidumbre a la aceptación de responsabilidades con uno mismo y con el otro. Las principales propiedades de la categoría son:
a) Inseguridad.
b) Necesidad de refuerzo docente.
c) Dificultad para gestionar cambios imprevistos.
d) Dispersión.

El reconocimiento de la permanencia en el tiempo del aprendizaje adquirido cooperativamente no parece incentivarles para superar el bloqueo ante la incertidumbre, a la luz de las entrevistas mantenidas varios estudiantes señalan sus dificultades para gestionar actitudes, cambios y comportamientos personales:

Cuesta asimilar que el profesor no va a estar permanentemente ahí guiándote (A).

Es importante que un profesor esté ahí, para ayudar a canalizar ideas y comportamientos del grupo, porque sino... te dispersas (E).

\section{DISCUSIÓN Y CONCLUSIONES}

A partir de la identificación de estas categorías, se generaron los esbozos de una teoría inicial sobre la percepción del uso cooperativo de las herramientas 2.0 para desarrollar un trabajo con tutorías entre iguales. Dichos esbozos se pueden resumir como sigue.

No es posible definir el potencial de estas herramientas y metodologías aisladamente del contexto y la finalidad, puesto que para los estudiantes entrevistados tiene significado diferente utilizarlas en el ámbito privado-personal, que en el ámbito académico, donde se ponen en juego sus calificaciones. Esto es, la variación en la percepción refleja el modo en el que los estudiantes conciben su utilidad y las competencias que le va a suponer conseguir un uso eficaz para el aprendizaje. 
La importancia que se le da al feedback entre compañeros y las competencias necesarias para que la relación sea formativa (motivación intrínseca, autoplanificación, autocontrol, habilidades sociales, espíritu de superación, saber hacer, saber estar, saber (dominio técnico), implicación, escucha activa, reflexión, pensamiento crítico, empatía, asertividad), dependen de las características personales del estudiante y del modo en que él mismo percibe la utilidad del trabajo a desarrollar y de las herramientas 2.0.

Al iniciar el desarrollo del trabajo, los estudiantes comienzan a ser conscientes de las limitaciones personales y técnicas en el uso de herramientas 2.0 que tienen y reaccionan ante ellas, solicitando compartir un peso relativo del trabajo con el profesorado implicado en el proyecto. Reconocen que la innovación y la recompensa motivadora es fuerte, puesto que se les ha dado la posibilidad de elegir cómo, dónde, cuándo y con quién desarrollar una actividad de tutoría y aprendizaje, verificando lo señalado por Collins y Moonen (2011). Igualmente la evaluación del proceso y producto de este trabajo cooperativo ha supuesto un acicate, puesto que la nota individual final fue la misma para todos los participantes, y tuvieron a su disposición, para superar las citadas limitaciones, a tres profesores y a ocho compañeros de titulaciones diferentes y afines a su título.

Los resultados obtenidos nos llevan a concluir que existe una percepción ambivalente respecto a la potencialidad de las herramientas 2.0 para desarrollar competencias socioprofesionales. Se considera que algunas herramientas son un complemento y un apoyo para desarrollar competencias, para debatir y elaborar un trabajo de forma inmediata, sin límites horarios o geográficos, y se diferencian como más exigentes a nivel competencial aquellas herramientas en las que no hay contacto visual -GoogleDocs/Drive, Twitter-, de aquellas en las que sí lo hay, como Google+, puesto que visualizar el lenguaje no verbal del compañero facilita el trabajo cooperativo. Se deduce así que son los compañeros del grupo los canalizadores del interés, el compromiso y la motivación por el trabajo y, no sólo las herramientas 2.0.

Las necesidades formativas que perciben sobre sus competencias socioprofesionales, en la línea de las pautadas por UNESCO (1996) y DeSeCo (2002), están relacionadas con el autocontrol ante la incertidumbre y la innovación -aunque no les motiva la rutina-, la autoplanificación del tiempo, el planteamiento de expectativas altas -no mínimas-, la búsqueda autónoma de documentación útil en la red, la reflexión y crítica constructiva, la gestión de actitudes y comportamientos de los otros y la confianza en sí mismos. Esto es así dado que reconocen depender en demasía de la norma, la regla, la guía o consigna de una figura a la que consideren experta, madura y profesional.

Nos parece interesante resaltar que mediante la realización de este proyecto de innovación se ha puesto de manifiesto el potencial de las herramientas 2.0, las tutorías entre iguales y el aprendizaje cooperativo en entornos virtuales. Entre las variables que han dado lugar a una conexión y un aprendizaje constructivo, resaltamos el lenguaje cercano en las explicaciones, la flexibilidad horaria y espacial para conectarse y trabajar con sus compañeros, coincidiendo con las apreciaciones encontradas por Hernando, Aguaded y Tirado (2011), la confianza para expresar dudas, contradicciones o problemas, la tolerancia, la asertividad y el deseo de compartir hallazgos con los compañeros. Aunque reconocen que se necesita un cambio de organización de horarios académicos, actualización permanente de recursos tecnológicos y metodología docente coherente con las competencias propuestas, para rentabilizar el esfuerzo que supone preparar las tutorías, dominar técnicamente el uso de las herramientas 2.0, coordinarse entre compañeros y con el profesorado, recibir un 
feedback formativo de ambos e incrementar el rendimiento y la calidad del aprendizaje, tal como afirman Fernández, Arco, Ortega y Heilborn (2011) y Alzate-Medina y Peña-Borrero (2010).

Por tanto, los resultados expuestos en este trabajo apuntan hacia la conclusión de que la tutoría entre iguales y el aprendizaje cooperativo en entornos virtuales permiten la superación de las limitaciones que los estudiantes encuentran para aprender a través de herramientas 2.0, y que la combinación de estos tres elementos hace posible la adquisición consciente de competencias.

Algunas propuestas de mejora de cara a futuras investigaciones irían en la línea de ampliar la aplicabilidad de este proyecto a otras áreas de conocimiento diferentes a las de Educación, estableciendo colaboraciones formativas virtuales entre docentes y estudiantes de otras titulaciones. Los resultados de este proyecto sugieren un análisis más amplio y profundo, tanto cualitativo como cuantitativo, sobre el grado y el tipo de implicación académica que los estudiantes universitarios están dispuestos a mantener si se les ofrece tiempo, seguimiento y recursos tecnológicos novedosos que les faciliten la búsqueda y transferencia de la información. Y, así también, se sugiere un análisis sobre la formación de las competencias demandadas como necesarias por los estudiantes universitarios.

\section{REFERENCIAS BIBLIOGRÁFICAS}

Álvarez, P. y González, M. (2005). La tutoría entre iguales y la orientación académica. Una experiencia de formación académica y profesional. Educar, (36), 107-128.

Alzate-Medina, G. M. y Peña-Borrero, L. B. (2010). La tutoría entre iguales: Una modalidad para el desarrollo de la escritura en la educación superior. Universitas Psychologica, vol.9 (1), 123-138.

Angell, L. C. \& Klassen, R. D. (1999). Integrating Environmental Issues into the Mainstream: An Agenda for Research in Operations Management. Journal of Operations Management, vol.17 (5), 575-598.

Arco, J. L. y Fernández, F. D. (2011). Eficacia de un programa de tutoría entre iguales para la mejora de los hábitos de estudio del alumnado universitario. Revista de Psicodidáctica, vol.16 (1), $162-180$.

Camilli, C., López Gómez, E. y Barceló, M. L. (2012). Eficacia del aprendizaje cooperativo en comparación con situaciones competitivas o individuales. Su aplicación en la tecnología: Una revisión sistemática. Enseñanza \& Teaching, vol.30 (2), 81-103.

Charmaz, K. (2005). Grounded Theory in the 21 st Century. In N. K. Denzing \& Y. S. Lincoln (Eds.), The Sage Handbook of Qualitative Research (pp. 507-535). London: Sage.

Collis, B. y Moonen, J. (2011). Flexibilidad en la educación superior: Revisión de expectativas. Comunicar, (37), 15-25.

Creswell, J. (1998). Qualitative Inquiry and Research Design. Chossing among Five Tradition. London: Sage.

DeSeCo (2002). Definition and Selection of Competences: Theoretical and Conceptual Foundations. Retrieved on March 21, 2013 from http://www.deseco.admin.ch/

Di Tommaso, K. (2012). Developmental Students: The Challenge of Peer Relationships. Community College Journal of Research and Practice, vol.36 (12), 941-954.

Duran, D. y Vidal, V. (2004). Tutoría entre iguales: De la teoría a la práctica. Barcelona: Graò.

Erstad, O., Gilje, O. y Arnseth, H. C. (2013). Vidas de aprendizaje conectadas: Jóvenes digitales en espacios escolares y comunitarios. Comunicar, (40), 89-98.

Evans, M. J. \& Moore, J. S. (2013). Peer Tutoring with the Aid of the Internet. British Journal 
of Educational Technology, vol.44 (1), 144-155.

Fernández, F. D., Arco, J. L., Ortega, S. y Heilborn, V. A. (2011). Prevención del fracaso académico universitario mediante tutoría entre iguales. Revista Latinoamericana de Psicología, vol.43 (1), 59-71.

Fernández Tilve, M. D. y San Juan, M. M. (2012). Entornos virtuales de aprendizaje: ¿Una ocasión para que nuestros estudiantes universitarios adquieran competencias profesionales? Edutec, Revista Electrónica de Tecnología Educativa, (42). Recuperado el 15 de enero de 2013 desde http:// edutec.rediris.es/Revelec2/Revelec42/

Glasser, B. G. \& Strauss, A. L. (1999). The Discovery of Grounded Theory: Strategies for Qualitative Research. New York: Aldine de Gruyter.

González Pérez, V., Traver Martí, J. A. y García López, R. (2011). El aprendizaje cooperativo desde una perspectiva ética. ESE: Estudios Sobre Educación, (21), 181-197.

Gros, B. y Romañá, T. (2004). Ser profesor. Palabras sobre la docencia universitaria. Barcelona: Octaedro-ICE Universidad de Barcelona.

Hernando, A., Aguaded, J. I. y Tirado, R. (2011). Aprendizaje cooperativo on-line a través del Campus Andaluz Virtual. Análisis de las interacciones. Enseñanza \& Teaching, vol.29 (1), 135-158.

Krueger, R. (2000). Focus Groups: A Practical Guide for Applied Research. London: Sage.

Lobato, C., Arbizu, F. y Del Castillo, L. (2004). Claves de la práctica de la tutorización entre iguales en las universidades anglosajonas. Algunas aplicaciones a nuestra realidad universitaria. Revista Enfoques Educacionales, vol.6 (1), 53-65.

Merton, R., Fiske, M. \& Kendall, P. (1990). The Focused Interview: A Manual of Problems and Procedures. London: Collier MacMillan.

Michavila, F. (2009). La innovación educativa. Oportunidades y barreras. Arbor. Ciencia, Pensamiento y Cultura, (185), 3-8.

Molina, P., Jimeno, L. A., Pérez-Samaniego, V., Devís-Devís, J. y Villamón, M. (2013). Uso de blogs y evaluación continua del aprendizaje del alumnado universitario. Edutec, Revista Electrónica de Tecnología Educativa, (43). Recuperado el 20 de marzo de 2013 desde http://edutec.rediris.es/ Revelec2/Revelec43/

Strauss, A. y Corbin, J. (2002). Bases de la investigación cualitativa. Medellín: Editorial Universidad de Antioquia.

Topping, K. J., Dehkinet, R., Blanch, S., Corcelles, M. \& Duran, D. (2013). Paradoxical Effects of Feedback in International Online Reciprocal Peer Tutoring. Computers and Education, vol.61 (1), 225-231.

UNESCO (1996). Learning: The Treasure Within. Report to UNESCO of the International Commission on Education for the Twenty-first Century. Paris: UNESCO.

Yuste, R., Alonso, L. y Blázquez, F. (2012). La e-evaluación de aprendizajes en educación superior a través de aulas virtuales síncronas. Comunicar, (39), 159-167.

Zeller, R. A. \& Carmines, E. (1980). Measurement in the Social Sciences: The Link between Theory and Data. Cambridge: Cambridge University Press. 